\title{
Towards a New Approach for Modelling Interactive Real Time Systems Based on Collaborative Decisions Network
}

\author{
Soumaya El Mamoune \\ Mathematical laboratory and Applications, ABDELMALEK ESSAADI University \\ Faculty of Science and Technology, Tangier, Morocco \\ E-mail: soumaya.elmamoune@gmail.com
}

Mostafa Ezziyyani

Mathematical laboratory and Applications, ABDELMALEK ESSAADI University

Faculty of Science and Technology, Tangier, Morocco

E-mail: ezziyyani@gmail.com

\begin{abstract}
Jaime Lloret
Department of Communications, Universidad Politécnica de Valencia

Camino Vera s/n, 46022, Valencia (Spain)

E-mail: jlloret@dcom.upv.es
\end{abstract}

Received: March 17, 2015

Accepted: April 23, 2015

Published: April 30, 2015

DOI: 10.5296/npa.v7i1.7257

URL: http://dx.doi.org/10.5296/npa.v7i1.7257

\begin{abstract}
Adaptive systems are being used in many research fields such as e-Learning, switching in the telephone network and for organizing contents on the Web. Each field requires a particular adaptation system which is not generally applicable to the other contexts. The lack of several criteria like the interaction with the environment, the self-improvement and the evaluation
\end{abstract}


pushed us to look further and suggest a generalized solution for any context. In this article, we present a new approach for the adaptation of the interactive systems of decision-making to offer suitable services to a given situation based on the interaction with the environment, the auto-improvement and the evolution of the system. The approach suggested is a graphic modelling called decisions network which will serve the design and construction of the dynamic operation of the core of the adaptive systems of decision-making. The service offered by such system is an adaptive final decision of the core which is a result got by the combination of the nodes of the decisions network and its iterative interactions via the environmental data. This work has two objectives: on the one hand, to present the new approach with its components and its importance by reporting existing work and, on the other hand, to present the approach with the chart and the formalization in order to model the dynamic behavior of the system via the search for a final decision in the form of adjacent elementary decisions.

Keywords: Adaptive system, auto-improvement, collaborative system, decision-making, graph of decision, interaction with the environment, modelling of the behavior, decisions network.

\section{Introduction}

One of the major challenges of the adaptive systems of decision-making aid is to present a complete approach of adaptation to the user's environment and the construction of an optimal and effective action plan. The need in this case is to analyze the treated situation and to make an adaptive decision which meets the needs of the user. Often a question arises, is it possible to perceive the changes of environment and to adapt them to the contexts of the user? In this direction, several researches were made and we found that it is not impossible but it is difficult by the classical techniques from which the idea is to propose a standard adaptation approach and decision making which ensures the best solution in a given situation. We propose in this article a new "Decisions network" approach which is able to adapt to a situation. This new approach facilitates the communication and managements the decisions in order to answer the needs with high quality services.

Our objective is to present the design and the organization of an adaptive decision-making aid system. We model and evaluate the system in order to show its dynamic structure and how it is able to adapt to the changes of its environment. Our approach has several interesting advantages such as the modelling and the visualization of behaviors, the evolution of the system, the synchronization and the scheduling of the decisions. On one hand, this article presents the studied problems and our motivation. Then, it presents the related work and discusses the limitations of each approach and the missing points. On the other hand, we present our contribution "Decisions network" with a well detailed architecture and finally we present its formalism.

The rest of the paper is structured as follows. In Section 2, we study the problem and motivation of the new approach by analyzing existing adaptive systems. In Section 3, we 
present the related work. We propose the new approach "decisions network" in section 4. We explain how we formalize the "decisions network", by citing the rules of decisions graph, in section 5. An example of decisions network application with simulation scenarios and the results of the discussion are shown in Section 6. Finally, we present the conclusion and proposals for future work in Section 7.

\section{Studied problems and motivation}

To conceive an adaptive system of decision-making aid in an evolutionary and changeable environment is a difficult objective to reach by using the classical approaches. In order to reach it, we take into account the quantity and variety of the received data, which also included the sensitivity of the field. We seek to conceive an adaptive system able to offer suitable services or solutions to the users containing their contexts. For example: a system of rescue which proposes to the user the way to be followed in the case of danger or general instructions to save him. Other example, an e-Learning system which offers support to a course or training for any user depending on its context, level, or indicator. This kind of system must collect and treat the information representing the context of users to offer them the best service. The service is a decision taken by the system in order to satisfy the users. It is an adaptive evolutionary system of decision-making aid which adapts to the context of users and helps them to take the best solution via its experimentation.

In the related literature, we find several adaptive systems. The problem is that each researcher proposes its own concept or his/her own proposal and we do not find an approach of common adaptation. The concept of adaptation is different for each case as each one defines the concept of the context according to its proposed system. So, the idea of our work is to present an approach of adaptation and decision-making aid standard which could be used for any adaptive system.

As shown in figure 1, the environment is represented by the environmental data. It communicates with the adaptive system to identify the context of the situation and propose the appropriate services. 


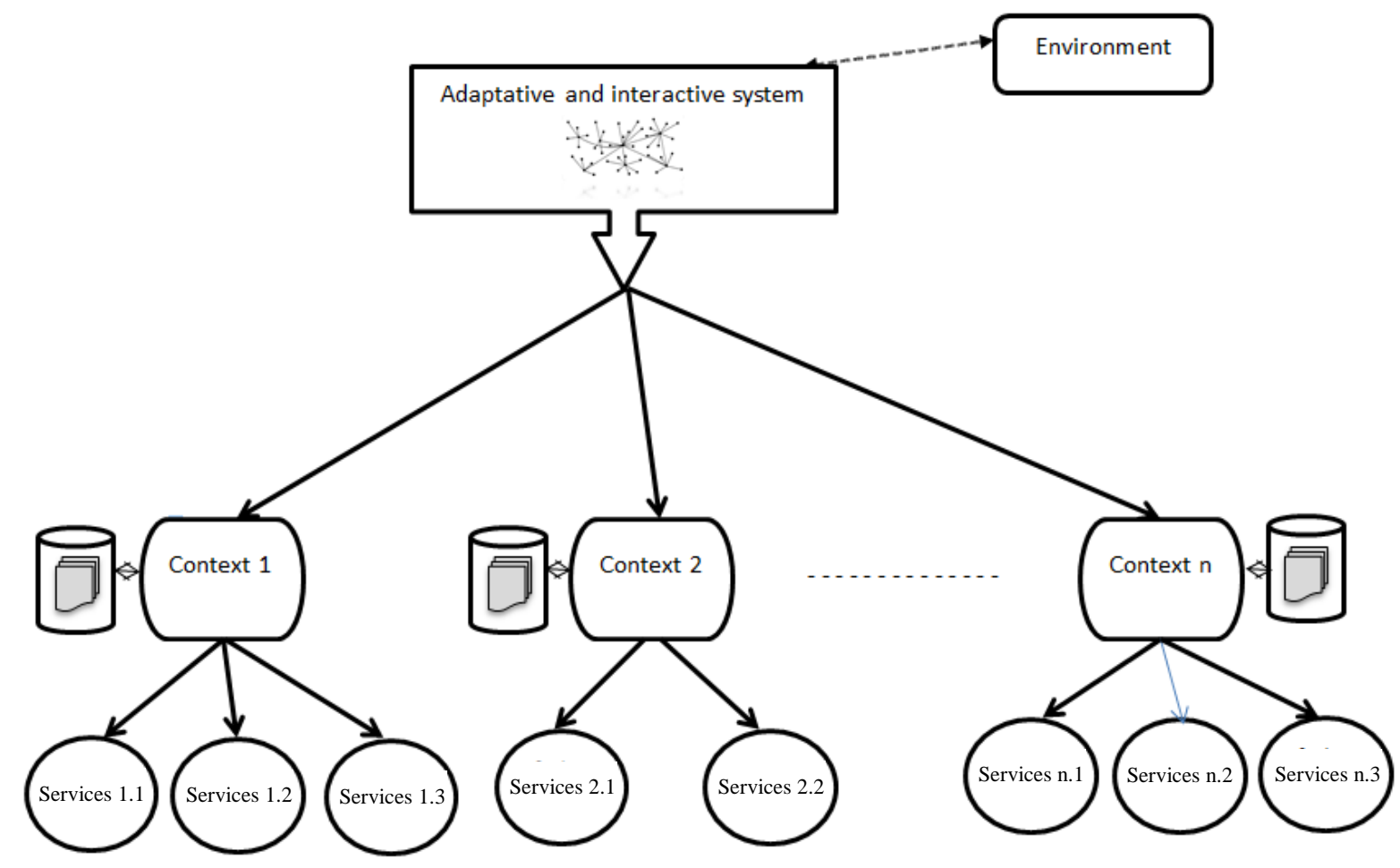

Figure 1. General architecture of the adaptive system

\section{Related work}

There are several published works and implemented applications about "adaptive systems". In this section we review some of them.

There is an adaptive system for e-Education, which was proposed by George, Gleizes and Glize in [1]. It aims at the search of trainers and relevant information sources starting from textual descriptions. To make it, the authors proposed an adaptive multi-agent system whose finality was to model the semantics of the field and it was based on the emergent organization.

We have also found an adaptive system of e-commerce in Internet [2]. It is an European project which if formed by partners having the objective to conceive adaptive software to improve this broking function. In this project, they proposed a system made of several self-organizing multi-agents systems of several levels. They included a role for each level.

Several researches have been made in order to suggest a solution of system adaptation in several fields such as teaching, tourism, medicine... Among this work, we can cite one of the famous ones: the AMAS theory and decision trees [1]. The adaptive multi-agent system, proposed in 2007, is an approach for the complex adaptive systems design, based on the adaptive multi-agent systems which seek to collaborate in order to provide a service or a decision. The collaboration of the agents, which was the objective of the theory, generates some problems between the agents. The major problem is the conflict in the situations where the agents have opposite proposals and the competition in the situations where the agents 
have similar proposals. This theory does not discuss an essential item which is the effectiveness of the proposed agents. The analysis of the quality of service allows the auto-improvement of all the system what pushes us to seek for another solution.

The decision tree is a decision-making approach which represents the more or less complex situation to which one must cope in the form of a tree where at the end of each branch are placed the various possible results according to the decisions taken in each stage. This approach is suitable with the adaptation and decision making. The processing of the received data, or the modification of a data, requires remaking all the tests starting from the first node of the tree (root). This repetition requires a very important computing time, but it is needed in order, to have a solution at the end. If the tree is large even it can negatively influence the system effectiveness. The decision tree is a popular training technique of adaptation but it is limited to complex situations [3] [4].

\section{Decisions Network}

To have a bad decision in a critical situation, such as natural disasters like floods, may generate unlimited damage in property and human damages. We find lot of researches proposing a system to notify the users [5], [6] or developing a decision support system for flood forecasting [7]. The main problem is having lot of systems, instead of an unique approach, for several disasters, which will imply to treat all the details and risks. This situation presents a challenge for the researchers and, in order to solve this need, we studied other system in other context. Concretely, we analyzed the organization of the information in the web [8] and the organization in the process workflow [9]. We found that the change of the environmental data is needed to assist the systems with their decisions. The decisions must tend at any moment towards a suitable function, which is not possible to do with the classical systems.

Because of the aforementioned reasons, our research aims to propose an approach which will be able to adapt to the environment in order to tend at any moment towards the adequate function. The suggested solution is a novel method to model the behavior of the adaptive system of decision-making aid, which makes the system to pertinently act in its medium according to its perceptions of the environment by using the sensors and its knowledge.

The suggested approach is called decisions network because a network indicates with the illustrated direction a set of relations. The decisions network is an inter-connected entity making components and its interrelationships to have an intelligent network by using artificial intelligence [10]. The decisions represent the services and they are inter-connected. They are used to model the dynamic of the adaptive and dubious system aiming at schematizing the behavior of the system.

\subsection{Motivation to develop a new approach}

In the related literature we find a lot of works to design the decision making system for disasters, like proposed in [11]. We found another work in [12], which presents a framework 
of a complex adaptive system of decisions. In these works, there is not an unique design of these solutions. So, we seek to propose an adaptation approach of unified decision-making aid which could be applied for the conception of an adaptive system to the context of users. The new approach must offer the suitable decision to the user by identifying his context taking into account the input data. After the analysis of the existing systems, we found that to achieve this kind of adaptation, it is necessary to propose a flexible approach. The approach could be applied to any adaptive system and it should not be fixed so that it can take into account each change and learn via its experiments.

\subsubsection{Interaction with the environment in real time}

The significant fields such as "the natural disasters" and "Medicine" need effective and fast special systems which carry out treatments dedicated to the needs. Several works have been published conceiving an adaptive system of decision-making aid that analyzes the information to provide an adaptive decision [13]. Most of these researches are based on the multi-agent systems, which provide results after the co-operation of the agents [1]. Most of them lack of an essential element of the adaptive system which is the interaction. This element allows the system to adapt to the environment and to check this adaptation every moment. Each change can influence the effectiveness and the output of the system. We verify of the solutions offered by the checking the satisfaction of the user's needs. The adjustment process will be started again until the system obtains the most suitable solution.

Figure 2 shows the implementation of our approach including the interaction with the environment at every moment. The adaptation uses the received data and takes into account the results for the previous adaptation. The interaction is necessary because we must have the situation-adaptive autonomy for time-critical takeoff decisions [14]. This point was an objective to be reached by our system which aims to take into account the environmental variables for the adaptation at every moment.

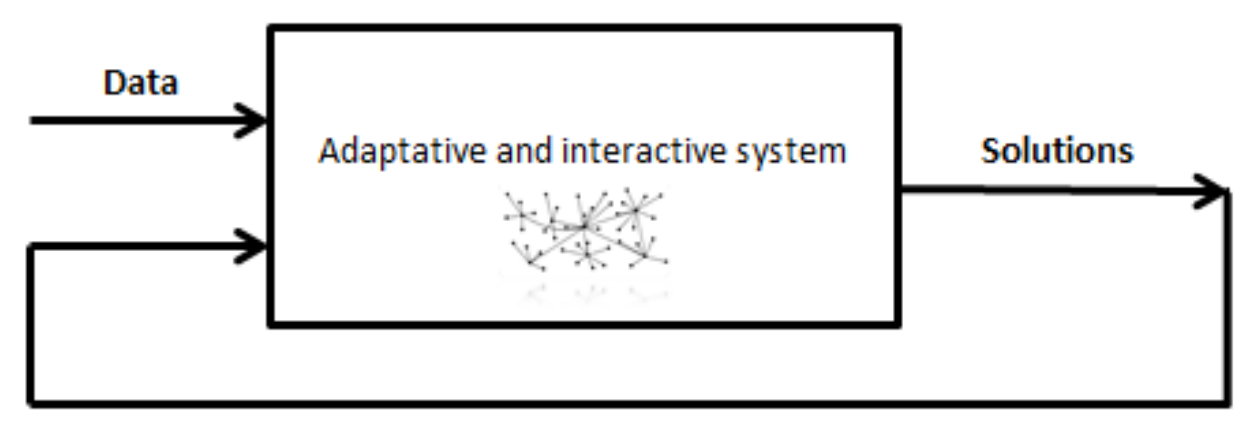

Figure 2. The interaction with the environment via the data

\subsubsection{System self-improvement}

To fix a service or to propose a lifetime action is a bad conception because everything is changing very quickly. Thus, we took into account this fact and we developed an essential 


\section{Macrothink}

point which is the system self-improvement, which is based on the experiments made by the system. The users' choice allows modifying a decision in order to increase the system effectiveness.

The experiments performed with the proposed system allow improving the quality of the solutions suggested, because they can be modified or removed if the solution is useless. After several turns, the user can notice that the decision given (D2) is an unusable decision or the most appropriate one was not selected as solution. For a given situation, we can modify the decision or replace it by another one as it is shown in Figure 3.

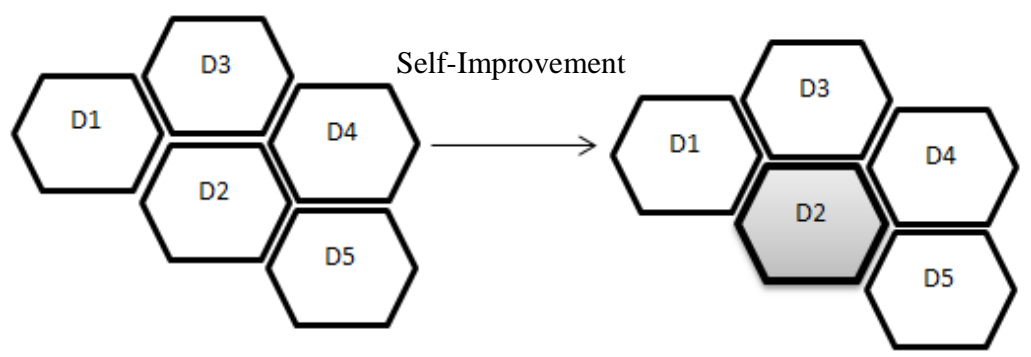

Figure 3. System self-improvement

\subsubsection{Evolution of the system}

The evolution is a basic criterion of our proposal. It is possible to evolve the system via its experiments by analyzing the data [15], deducing the probability to have a specific decision and extracting new knowledge which could be applied to the next uses. The system is empty at the beginning and it is created by gradually building its own ontology of the field via its knowledge and developing more and more the services. It is necessary that the memorized knowledge evolves in an automatic way. In the course of operation, it is not needed the intervention of the human user to have an evolutionary system. We also used the learning technology, which was used to have sequential decision making [16].

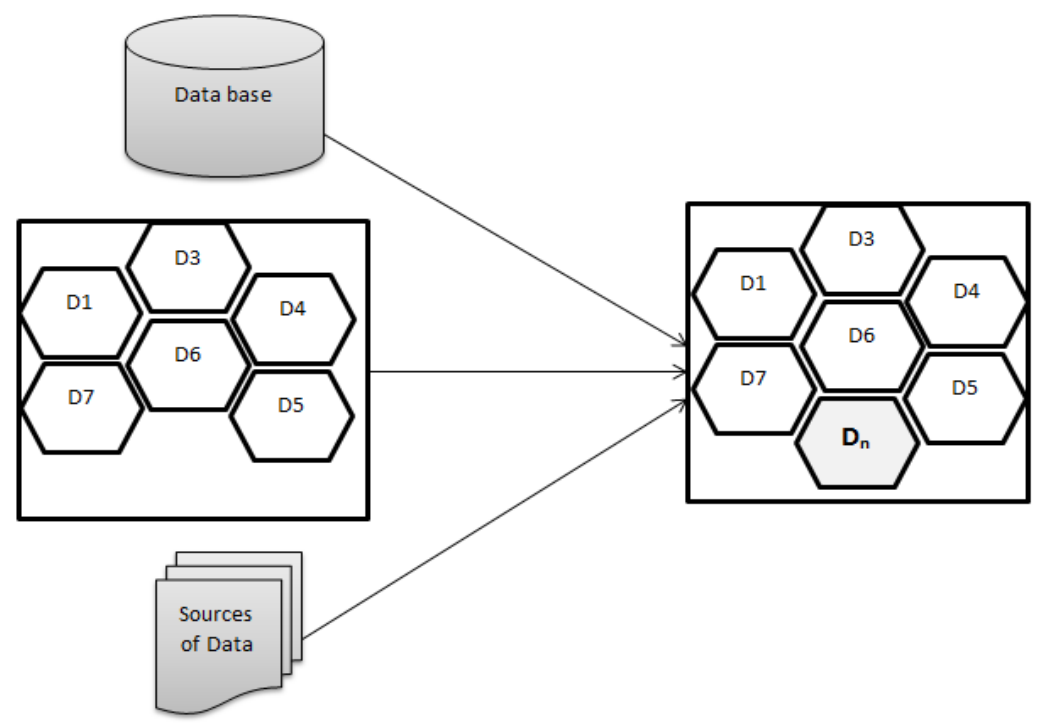

Figure 4. Construction and evolution of decisions network 
As it is shown in Figure 4, the elementary decisions are created gradually starting from the experiment of the system, which information is stored in a different data source (Database, ontology trees, files...). They build a more solid core.

\subsection{Principle of decisions network}

The decisions network is an approach of decision making which can be used by any system of decision aiming at offering an adaptive decision to the user context. The idea is to structure the services offered by the system in the form of a network which includes a succession of elementary decisions dependent between them according to the rules and the needs of the operating of the system.

The final decision offered by the system is a combination and regrouping of the elementary decisions which represent partial services to offer. We graphically structured the elementary decisions in the form of a graph of dependence where the decisions network is built starting from the knowledge of the system to model the dynamic behavior in a given situation. Memorized knowledge evolves in an automatic way and in the course of operation without requiring a great intervention of the human user to have an evolutionary system of decision.

The proposed protocol operates as follows. The data is sent to the server continuously. The system verifies the data and uses the information gathered from the sensor in order to identify whether there is a disaster or not. In case of a disaster, the system locates the user and the server sends the user the services according to its situation. The message flow is shown in Figure 5.

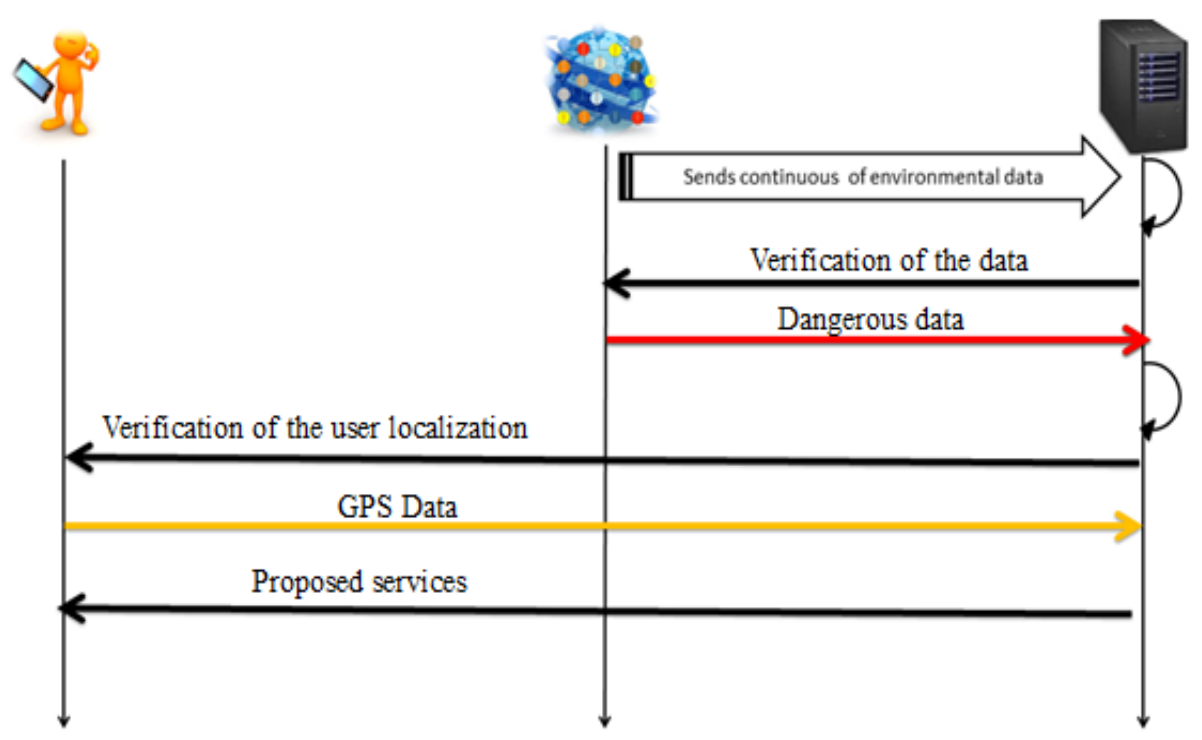

Figure 5. Communication Protocol

The sequence flow diagram of a regular case using our system is shown in Figure 6. It presents the communication and adaptation steps that have been taken into consideration to develop our approach. Sensors are placed in the environment. They send the environmental data. The system verifies if these data is dangerous. When the data is dangerous, the system verifies the situation and localizes the users using the GPS data. If the user is near the place 
of danger, the system notifies the user by sending him/her a message and proposes the way to avoid it. But if the user is in danger, the system proposes some services to solve it.

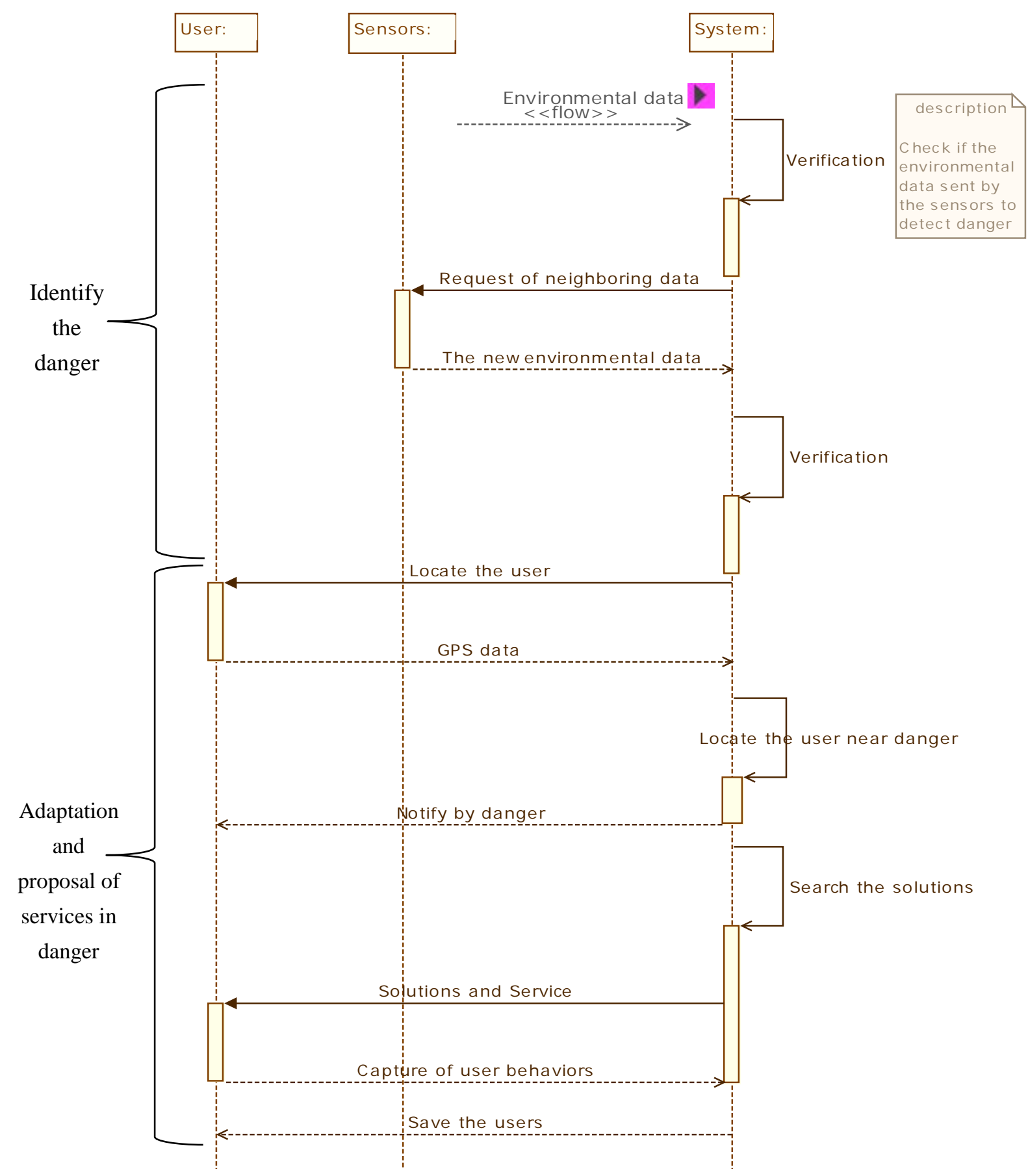

Figure 6. Sequence diagram

The sequence diagram follows the algorithm provided in figure 7 . The sensors send the environmental data to the system and the system verifies the disaster by the request of the neighboring data. After the verification, the system locates the user using the GPS data and notifies him the danger and proposes the solutions or the services to save him. Then we capture the users' behaviors to propose the adequate services. 


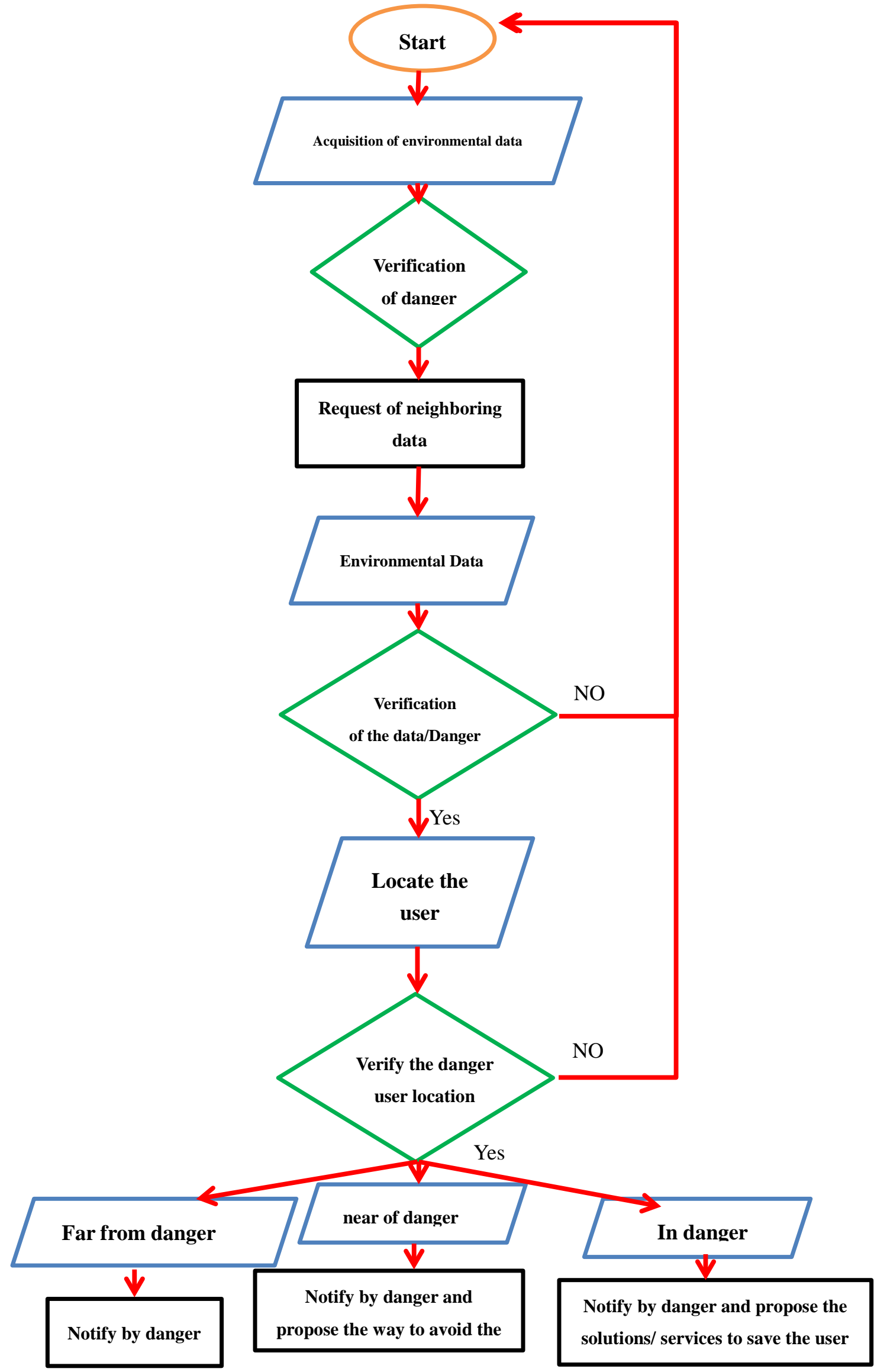

Figure 7. Proposed Algorithm 


\subsection{General example of the decisions network}

We present the graph with all its components in Figure 8. They are the elementary decision $(1,2,24,8,10,7 \ldots)$, the various connectors $(11,17,18)$ and the arc that connect the elementary decision and the connectors. The elementary decisions that have the same context are grouped in partial decision.

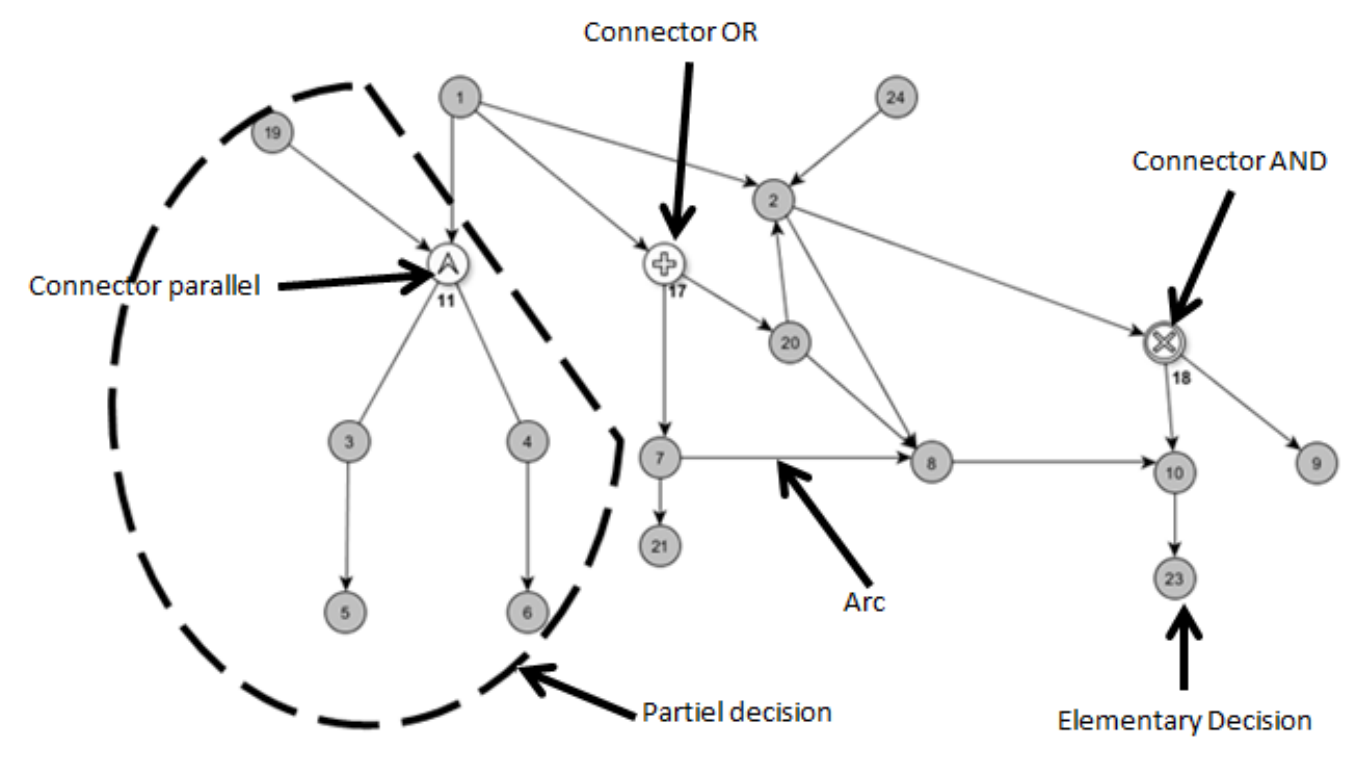

Figure 8. Example of decisions in the network

\section{Formalization of the decisions network: Decisions graph}

In this part, we define the formalization of a new approach entitled graph of decision allowing the resolution of the complex problems in the dubious fields by the automatic adaptation and the proposal of the services to be returned.

We begin this part with the definition of the rules and the constraints of construction of the Representation of Decisions (RD) network. Then, we define the presentation of the standards proposed by us and the chart and matrix for a good performance. Finally, we show the validation of our approach.

\subsection{Rules of construction of a RD network}

It must follow next rules:

- $\quad$ Each node has at least a data of activation which represents the event release.

- $\quad$ Each node has at least one result.

- Each node must be dependent to another node of the network at least (it cannot be isolated nodes).

- A connector must be limited by two nodes.

- There exist several types of connectors and, according to the type of execution, a 
node chooses the connector.

- $\quad$ The arc is the link which connects two successive nodes.

- Each arc has a cost which represents the degree of complementarity, compatibility and dependence of the two nodes.

\subsection{Representation of the decisions network}

\subsubsection{General standards}

The graph proposed by us uses the standard notion of the directed graphs besides the new notations in order to formalize the new features of the decision graph proposed for the the adaptive system. The decision graph is defined by the triplet GD $=\left\{<N_{\text {in }}, C, N_{\text {out }}>\right\}$ whose elements are $\mathrm{N}_{\text {in }}$ (the node of entry), $\mathrm{N}_{\text {out }}$ (the node of exit) and $\mathrm{C}$ (the connector connecting the nodes realized by an arc) and, graphically, it is presented as shown in Figure 9.
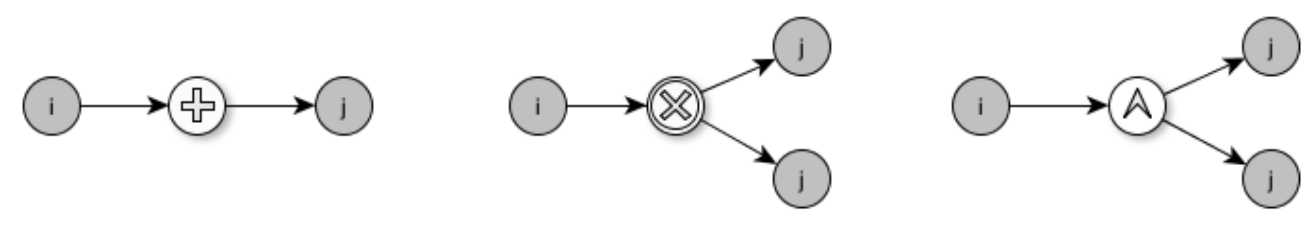

Figure 9. Various triplets of the graph

We call cost of an arc, named as C, the degree of complementarity, compatibility and dependence of the two nodes. It is presented in the form of a probability. It is calculated starting from the parameters of each decision. We define the cost using formula (1):

$$
C=\left(\sum_{i=0}^{n}(\operatorname{Pmin} / \mathrm{Pmax})\right) / N
$$

Where:

- $\quad \mathrm{N}$ is the number of common parameters.

- $\quad \mathrm{P}$ is the common parameter between the nodes.

- $\quad \mathrm{P}_{\min }$ is the minimal value of a common parameter.

- $\quad \mathrm{P}_{\max }$ is the maximum value of a common parameter.

\subsubsection{Graphical representation of the decisions network standard}

As it has been already shown, we represent our decisions network as the graph defined above. In figure 10, we show the decisions graph by the representation of the elementary decision and the connectors. 


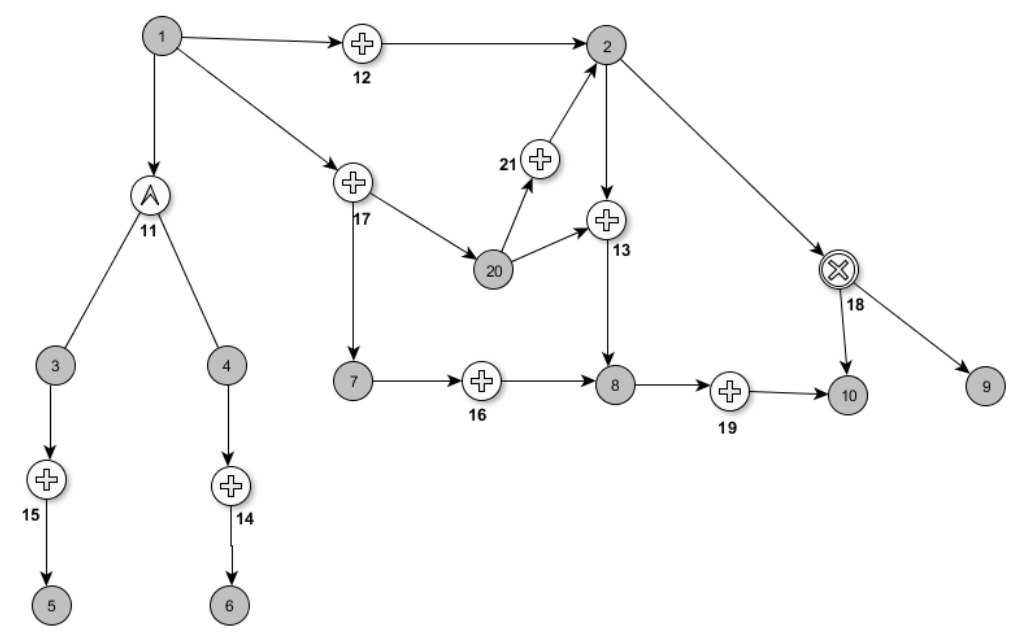

Figure 10. Example of graphic representation of graphic RD

For a simpler representation, we can simplify the graph by removing the simple connectors, which are the connectors connecting two successive nodes. The result is shown in figure 11 . We have removed the simple connectors like the connectors $12,14,15,16,19$, and 21, and we keep the essential connectors that cannot be eliminated.

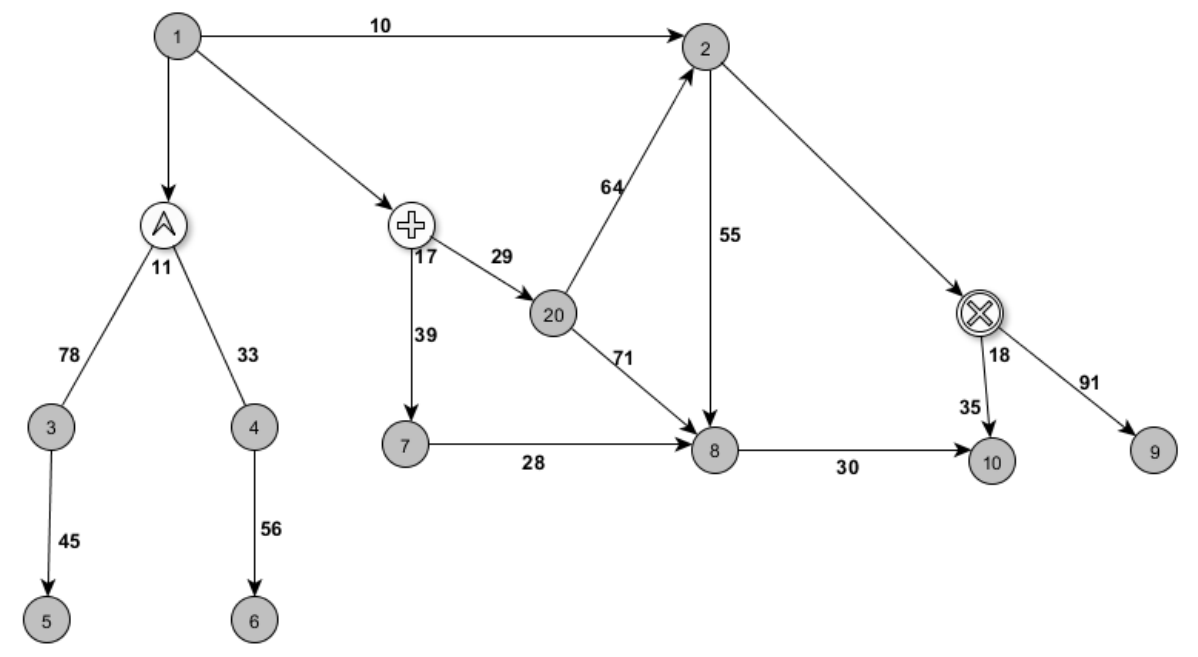

Figure 11. Example of refined graph RD

\subsubsection{Matrix representation of the decisions network}

Our decisions network RD can be represented in the form of a matrix. We call it MRD. MRD $(m \times n)$ is a table of $m$ lines and $n$ columns. $(I, J)$ indicates the intersection of line $i$ and the column j. In MRD, the lines and the columns represent the nodes of the graph.

For a given graph, there exists only one stamp which changes according to following rules:

The matrix is square. It has as many lines as columns. Next we highlight its main features: 
- The dimension of the matrix $(\mathrm{N})$ is the sum of the nodes and the connectors of the graph.

- $\quad$ Each node is identified by a single identifying code.

- Each connector is identified by a single identifying code.

- The arc connecting two nodes in the refined graph is represented by its cost.

- $\quad$ Each element of the matrix is characterized by $\mathrm{M}(\mathrm{I}, \mathrm{J})$. $\mathrm{I}$ is the number of line and $\mathrm{J}$ is the number of column. M(I, J) value is the cost of the arc connecting element I and element $\mathrm{J}$.

- The diagonal M(I, I) represents the type of element I and it contains obligatorily a value:

$\checkmark \mathrm{M}(\mathrm{I}, \mathrm{I})=0$ : element $\mathrm{I}$ is a node (elementary decision)

$\checkmark \quad \mathrm{M}(\mathrm{I}, \mathrm{I})=1$ : element $\mathrm{I}$ is a connector of the type or

$\checkmark \mathrm{M}(\mathrm{I}, \mathrm{I})=2$ : element $\mathrm{I}$ is a connector of the type and

$\checkmark \mathrm{M}(\mathrm{I}, \mathrm{I})=3$ : element $\mathrm{I}$ is connector of a parallel type

$\checkmark \mathrm{M}(\mathrm{I}, \mathrm{J})=-1$ : no relations between element I and $\mathrm{J}$.

- The arc connecting a node and a connector with several relations of the nodes will be represented by the maximum of the following arcs.
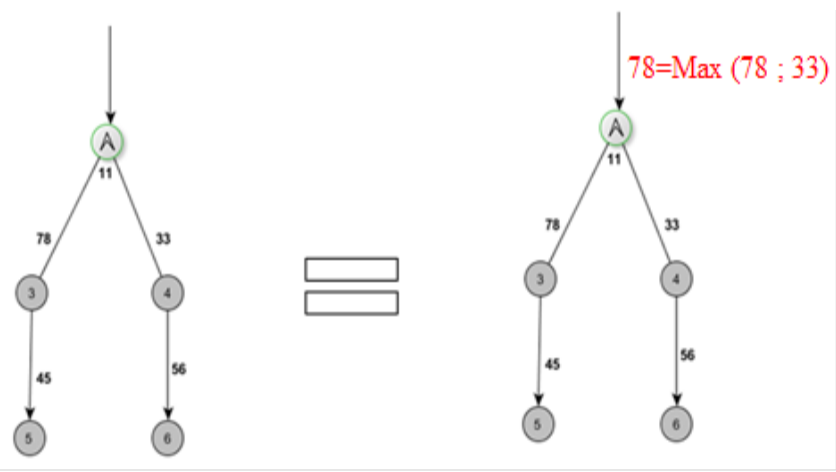

Figure 12. Cost calculation of the arc connecting a connector with several branches

We observe the rules presented previously to build the matrix of the graph presented above. The graph is constituted of 14 elements (11 elementary decisions and 3 connectors) then we create a matrix of 14 lines and 14 columns and we pass to the filling boxes of the matrix. 


\begin{tabular}{|c|c|c|c|c|c|c|c|c|c|c|c|c|c|c|}
\cline { 2 - 16 } \multicolumn{1}{c|}{} & 1 & 2 & 3 & 4 & 5 & 6 & 7 & 8 & 9 & 10 & 11 & 17 & 18 & 20 \\
\hline 1 & 0 & 10 & -1 & -1 & -1 & -1 & -1 & -1 & -1 & -1 & Max & Max & -1 & -1 \\
\hline 2 & -1 & 0 & -1 & -1 & -1 & -1 & -1 & -1 & -1 & -1 & -1 & -1 & Max & -1 \\
\hline 3 & -1 & -1 & 0 & -1 & 45 & -1 & -1 & -1 & -1 & -1 & -1 & -1 & -1 & -1 \\
\hline 4 & -1 & -1 & -1 & 0 & -1 & 56 & -1 & -1 & -1 & -1 & -1 & -1 & -1 & -1 \\
\hline 5 & -1 & -1 & -1 & -1 & 0 & -1 & -1 & -1 & -1 & -1 & -1 & -1 & -1 & -1 \\
\hline 6 & -1 & -1 & -1 & -1 & -1 & 0 & -1 & -1 & -1 & -1 & -1 & -1 & -1 & -1 \\
\hline 7 & -1 & -1 & -1 & -1 & -1 & -1 & 0 & 28 & -1 & -1 & -1 & -1 & -1 & -1 \\
\hline 8 & -1 & -1 & -1 & -1 & -1 & -1 & -1 & 0 & -1 & 30 & -1 & -1 & -1 & -1 \\
\hline 9 & -1 & -1 & -1 & -1 & -1 & -1 & -1 & -1 & 0 & -1 & -1 & -1 & -1 & -1 \\
\hline 10 & -1 & -1 & -1 & -1 & -1 & -1 & -1 & -1 & -1 & 0 & -1 & -1 & -1 & -1 \\
\hline 11 & -1 & -1 & 78 & 33 & -1 & -1 & -1 & -1 & -1 & -1 & 2 & -1 & -1 & -1 \\
\hline 17 & -1 & -1 & -1 & -1 & -1 & -1 & 39 & -1 & -1 & -1 & -1 & 1 & -1 & 29 \\
\hline 18 & -1 & -1 & -1 & -1 & -1 & -1 & -1 & -1 & 91 & 35 & -1 & -1 & 3 & -1 \\
\hline 20 & -1 & 64 & -1 & -1 & -1 & -1 & -1 & 71 & -1 & -1 & -1 & -1 & -1 & 0 \\
\hline
\end{tabular}

Figure 13. Matrix resulting from the graph

The decisions network is represented graphically by a graph and practically by a matrix which will allow the application of the algorithms.

\section{Example of an application of decisions network}

The objective of this section is to present a case study where a user can use the solution suggested by the system. It allows us to show how the system can adapt to a situation and offer the suitable services. Our solution is applied to the management of natural disasters and, concretely, to the fire. Finally, it is necessary to specify the degree of gravity and the solutions to be proposed to the users who are in danger in order to save their lives.

Before launching the modelling process, the system gathers the observations received, that is, the data sent by the sensors and/or the data sent by a user. The received data can be an event release of the process of adjustment which has been detected as abnormal. For our case if the temperature is higher than $90^{\circ}$ the value is an index of fire which causes the launching of the decisions network.

The addition of the elementary decisions and the precision of the events releases are made with the assistance of an expert or with the experiment of the system. Now, we present some decisions that are going to be used in this case study:

- To locate the sensor: Once the data is received, it is necessary to locate the sensor to locate the danger and, with this intention, it is necessary to relate sensor with the network of the sensors. This checking is a condition "Pre_condition" that the system will check before the execution of the task. The sensor must be equipped by a GPS or its placement must be registered in a database. This information is also a condition "Pdt_condition" and it is necessary to ensure that this sensor will send by means of any type of communication technology (WiFi, cable...).

- To collect the temperatures sent by the closest sensors: The same aforementioned 


\section{Macrothink}

conditions must be applied to the remaining sensors.

- Other decisions:

- To collect the speed of the wind

- Nature of the location

- To locate the users close to the danger

- To propose the way to be followed

- To inform the persons in charge

- To check the state of the user

\section{Case study}

We seek to apply our new approach to a real case study. It is flooding and to properly simulate the treatments we have chosen an example given in figure 14. This figure shows the region where we perform the study. In this area, several sensors are deployed in order to capture the environmental data.

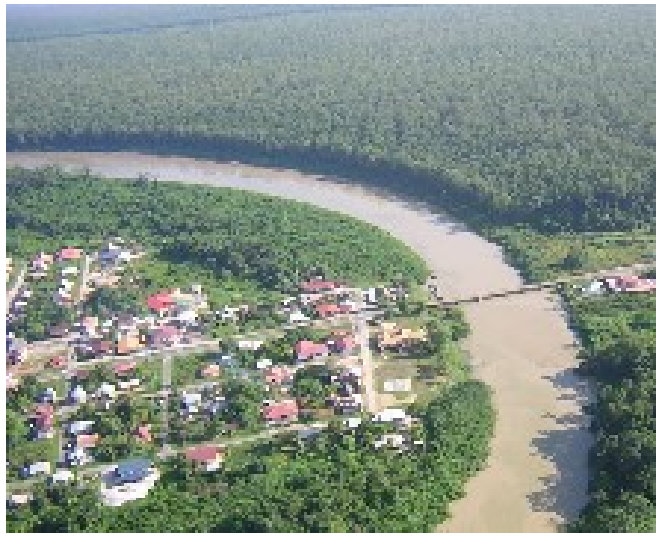

Figure 14. Image of a selected region to simulate the approach

The scheme shown in figure 15 is done according to figure 14. It shows the map of the region and shows the position of the sensors deployed.

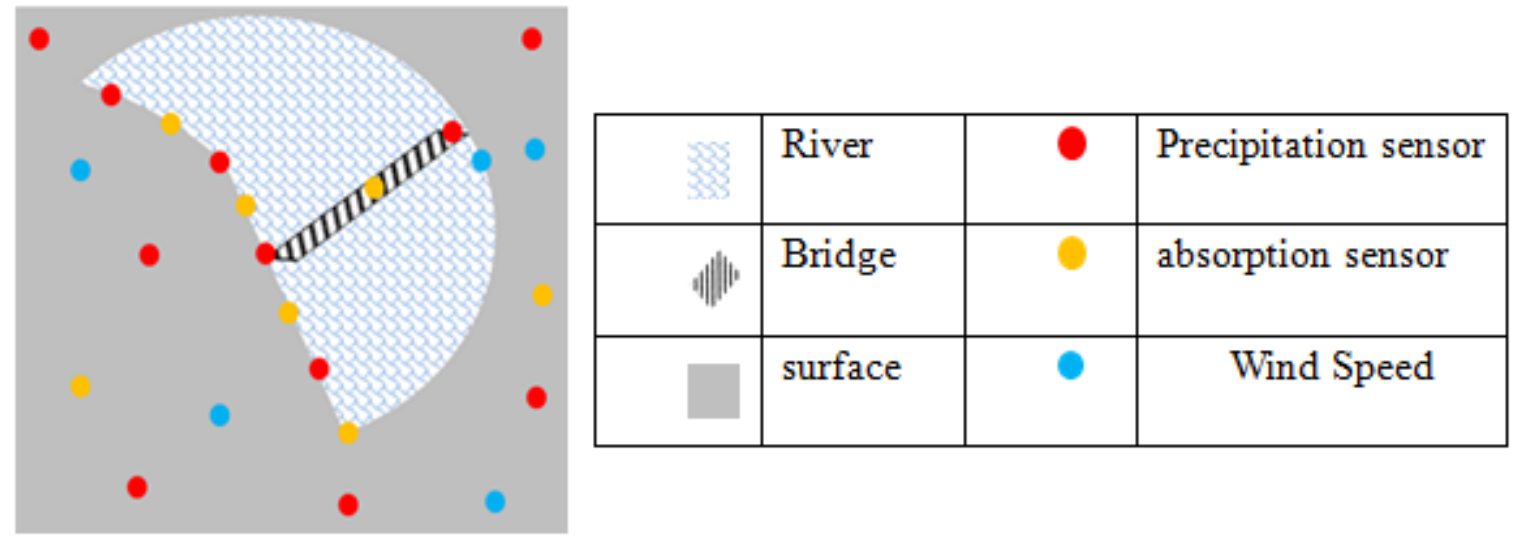

Figure 15. Schematic of the study area. 
According to the environmental data and the structure of the region, we choose the right decision to be an evacuation, support or rescue with ambulances, plane, boat or other means. The choice of the rescue services to be provided will vary according to the parameters sensed by the sensor network.

Generally, the parameters to capture and analyze are specified by experts. Moreover, they let us know which values are regular and which values are harmful. For this case study we chose four main environmental data: temperature, precipitation, absorption and wind speed. These parameters allow us to identify both the disaster and the type of service to be provided.

The parameters are analyzed according to the thresholds, which must not exceed, but in case of exceeding the thresholds, an alert will be generated. The importance of our approach is the detailed analysis of these values with the generation of good warning, instead of generating false alarms, thus we will reduce false alarms. These are the thresholds for this case:

- Temperature: It is supposed to do an intervention if the temperature is lower than $-3^{\circ} \mathrm{C}$ or higher than $40^{\circ} \mathrm{C}$. Moreover, the system will remove values that are not logical.

- Wind speed: According to the Beaufort scale, which is an empirical measurement scale with 13 degrees (0 to 12), the average wind speed is captured over a period of five minutes. We used this scale because it is effective in maritime environments and Beaufort number corresponds to a state of the associated sea at a "fork" of the mean wind speed.

- Absorption: This is the parameter that identifies the degree to suck water through the channels.

- Precipitation: the quantity of water falling in a specific area within a specific period. The difference between the precipitation and the degree of absorption can identify the flood. The precipitation is measured by millimeters "mm": $60 \mathrm{~mm}$ is represented by 60 liters of water per square meter.

- Water level: This is the volume of water falling on the more or less large area. It is also the amount of water flowed on the surface or in the study area.

Then, the system process the data collected from sensors in order to identify the disaster and the services to be performed.

The analysis is an essential step that eliminates false alarms. Generally, alerts are generated by comparing the received value with the thresholds and if the received data exceeds the maximum value, an alert is generated, but at the same time we must verify the reality of the data for not having false alarms.

Our goal is therefore to minimize the high number of false alarms and this can be done by using the collective decision between elementary decisions, which use the principle of decisions network operation.

In the table of values of the parameters used to control this region, we see several false alarms in a small interval. Concretely, there are 8 false alarms in temperature and this number 


\section{Macrothink}

Network Protocols and Algorithms

ISSN 1943-3581

2015, Vol. 7, No. 1

is quite higher when there more parameters. But if the network is using decision making, we will have the opportunity to verify and validate alerts and reduce false alarms.

Analyzing Figure 16, we see that the temperature increases along the time. We also see that there are four anomalous values and discontinuities, so, in this case, four false alarms will be generated. Using our approach, and after cooperation decisions, these false alarms will be eliminated because it does not represent real values.

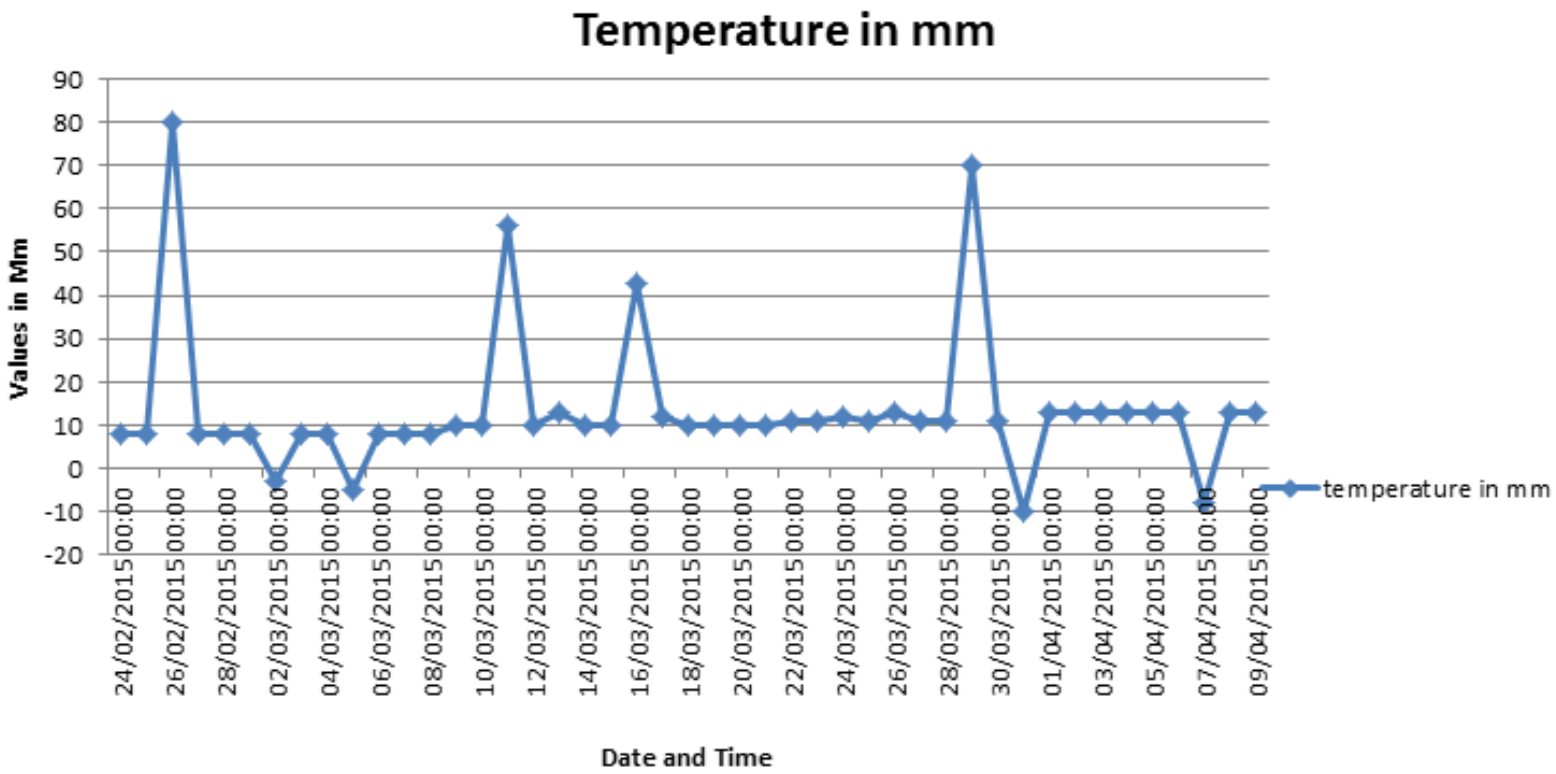

Figure 16. Graphical representation of temperature values

The identification of the disaster will not be effective until the precipitation and absorption are gathered in order to estimate the amount of water that circulates. The data gathered is shown in Figure 17.

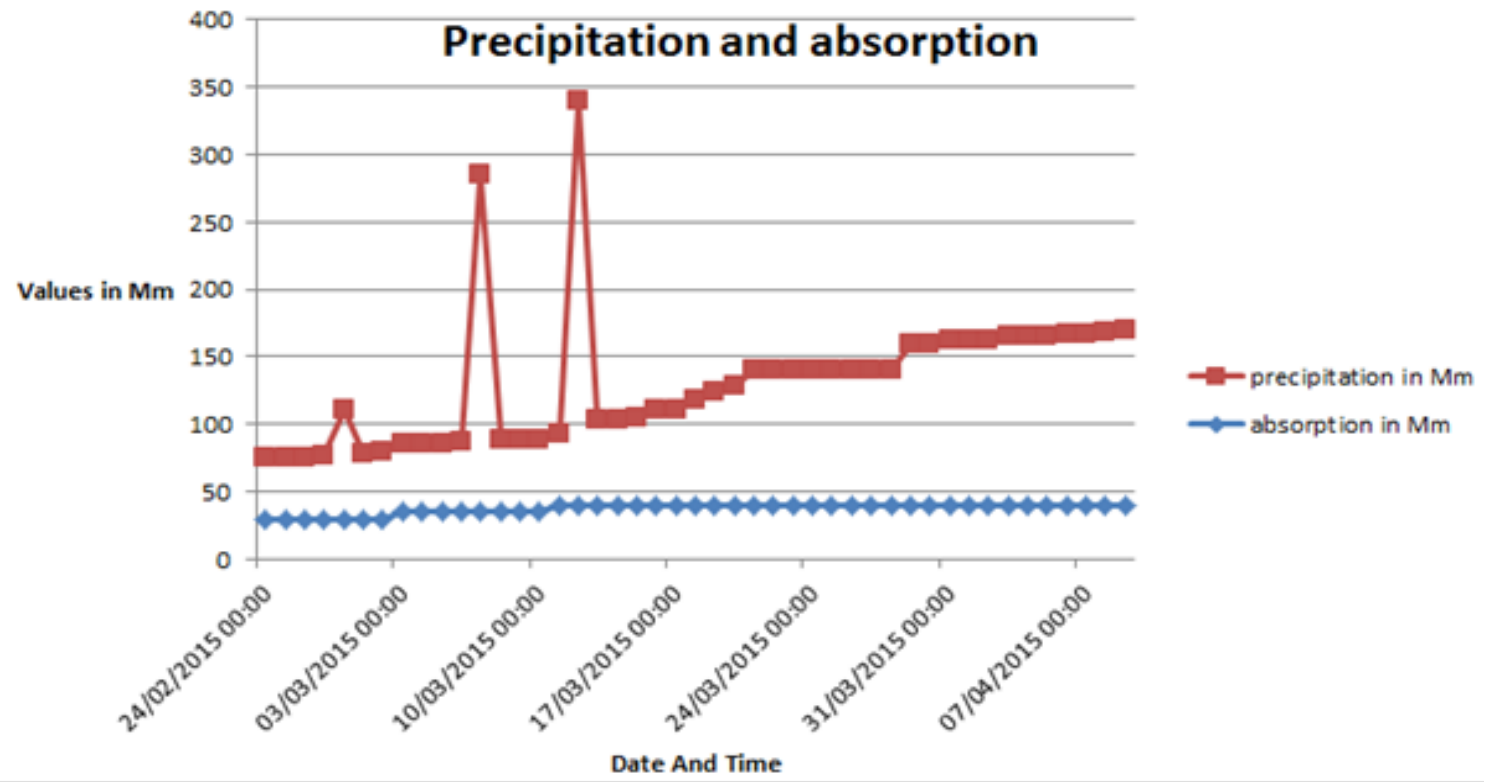

Figure 17. Graphical representation of the precipitation and absorption 
In figure 17, we observe a difference between the two parameters. This difference let us conclude that there is no flooding, but if we notice that there is a great difference between the average of the two parameters, because the water is not sucked, a warning will be generated.

Figure 18 shows an example where the precipitation exceeds the critical level during a time interval. Thus, there will be an alarm. But taking into consideration that the flow of absorption exceeds the precipitation rate with good water drainage procedure, the alert will be considered a false alarm. In this case, if we consider the separate treatment of data in different settings, there will be a very high number of false alarms that will negatively influence the quality and safety of the system. Another issue concerning the separate treatment of the parameters is the right choice of actions for intervention. When there is a disaster like a flood, given due to a strong precipitation, if we do not consider the water level and the wind speed, the decision making system will not be efficient. The collective processing of all parameters via the decisions network will determine the exact situation and a global vision for an adequate decision.

Figure 18 shows a clearer vision of the individual parameters analysis. For example, when analyzing the water level we can see that there are four false alarms because four values exceeded the critical level. The combination of the three parameters and the adjustment values received is the effective solution offered by our new approach. Figure 18 let us have a big picture by grouping three parameters, which let us provide an adequate decision.

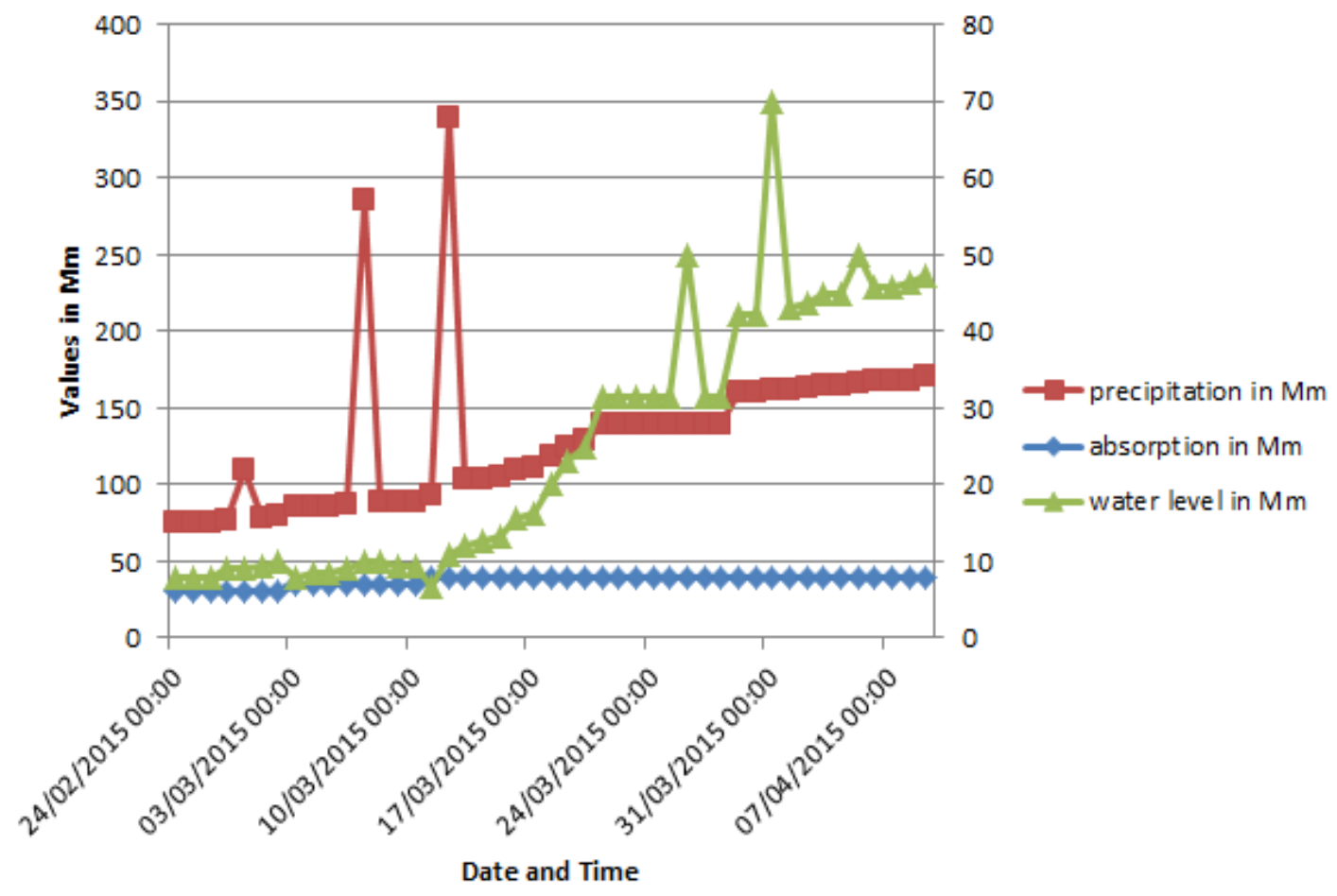

Figure 18. Graphical representation of the precipitation, absorption and water level

The services are the list of decisions to be taken for a rescue. As we have already presented, the rescue is done by several techniques and the right decision is to choose the right technique taking into account the environmental data. 
Let us suppose that we have the following decisions:

- Support: these are donations to support people in times of need.

- Rescue with ambulance: saving people and sending them to the hospital providing the fastest and safest path to the hospital.

- Rescue with boat, helicopter or aircraft: save people by sending them to the other side, knowing the climate and atmospheric conditions, will ensure the security of the transport.

Figure 19 shows three false alarms. Analyzing the data received, the system can deduce that the rescue by air can only be done if the wind speed is below the maximum value (in figure 19 we can see only two peaks over the maximum wind speed value).

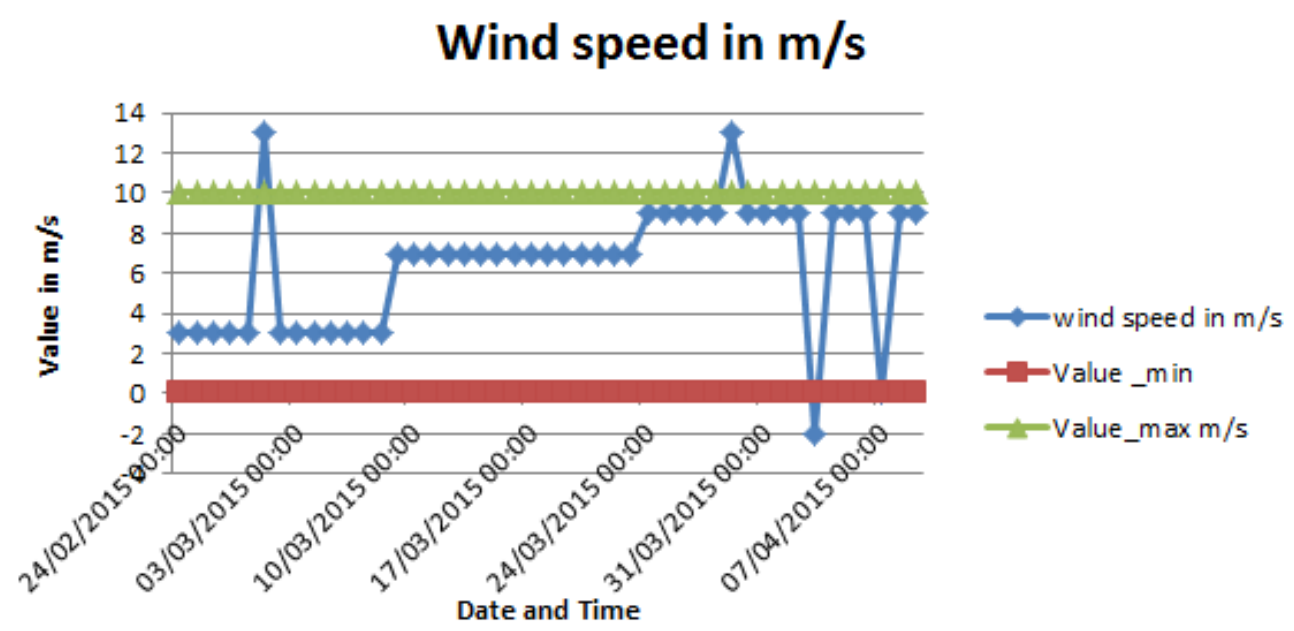

Figure 19. Graphical representation of the wind speed

\section{Conclusion}

To make a good decision, multiple conditions should be met. To specify the most accurate decision, the user profile should be taken into account. It can act in a simple decision or a more or less coherent whole of dependent decisions. The whole procedure should be determined, during and afterwards, to be sure of the good execution of the actions. The process must indicate the right options, which can be subjected to the process (input data: event release) and which can leave there (output: conditions and results).

The decisions network is the solution suggested for modelling complex adaptive systems, which is based on the interaction with the environment or the user, the self-improvement and the evolution. The procedure suggested allows the evolution of the final decision by taking into account the limits presented in the AMAS theory and the decisions trees. We sought to solve with this proposal to present a general decisions network architecture. We developed algorithms which assign a function to each elementary decision and we have defined the principles of connectors and arcs.

In future works, we will define more algorithms to manage the decisions network in 
order to ensure the communication with different entities from the system. Moreover, we will propose a new language that will allow us to manipulate the decision of the network. We are also planning to develop a database tostore the decision graph and services in different situations.

\section{Acknowledgment}

This work has been supported by the "Ministerio de Economia y Competitividad", through the "Convocatoria 2014. Proyectos I+D - Programa Estatal de Investigación Científica y Técnica de Excelencia" in the "Subprograma Estatal de Generación de Conocimiento”, Project TIN2014-57991-C3-1-P.

\section{References}

[1] Jean-Pierre George, Marie-Pierre Gleizes, Pierre Glize, "Conception de systems adaptatifs à fonctionnalité émergente : la théorie Amas” (2007). Revue d'intelligence artificielle, ISSN 0992-499, vol. 17/4 - 2003, pp. 591-626. http://dx.doi.org/10.3166/ria.17.591-626

[2] Maya Rupert "Organisation du contenu du Web selon la perspective des Systèmes Complexes Adaptatifs”. CORIA'2006(COnférence en Recherche d'Informations et Applications), ARIA de Lyon France. pp. 283-294.

[3] EL Mamoune S., Ezziyyani M. (2013). Adaptive Learning in the Simulation Of Emergency. In 3rd International Conference on Information Systems and Technologies (ICSIT’13) March 22-24, 2013, Tangier Morocco.

[4] EL MABROUK Marouane, KASSARA Omar, EL MAMOUNE Soumaya, EZZIYYANI Mostafa, ESSAAIDI Mohammad, GAOU Salma, ABAJJA Amal, “An Adaptive Intelligent Decision Support System for Real-Time Flood Forecasting”, International Journal of Research in Computer Applications and Robotics. Vol. 1, Issue 7, pp. 14-23, October 2013.

[5] Manavalan, Subrata Chattopadhyay, Mangala, Yalamanchili S.Rao, "Emerging trends of Computational Grid based Near Real Time/Real Time Flood Assessment and Forecasting Models", in Proceeding of the Third International Conference on Emerging Trends in Engineering and Technology" November 9- 11, 2012, Kurukshetra, Haryana, India, pp. 471- 475, http://dx.doi.org/10.1109/ICETET.2010.54

[6] Amjad RATTROUT, Taroub ISSA, Hassan BADIR, "Intelligent Web system: Using Intelligent Web Agents to Achieve a Self-Organized System" IEEE Conf SKIMA09 Fes, Morocco, October 21-23, 2009. pp 93-101

[7] Jin Wang, "Development of a Decision Support System for Flood Forecasting and Warning - A Case Studyon The Maribyrnong River”, PhD thesis, Victoria University, Melbourne, Australia, January 2007.

[8] Eirinaki M., Lampos C., Paulakis S., Vazirgiannis M., "Web personalization integrating 
content semantics and navigational patterns", Proceeding of the sixth ACM CIKM international workshop on web Information and Data Management, November 05 - 11, 2006, Washington DC, USA. pp 72- 79. http://dx.doi.org/10.1145/1031453.1031468

[9] Lotfi Bouzguenda, Manel Turki "Designing an Architectural Style for Dynamic Medical Cross-Organizational Workflow Management System: An Approach Based on Agents and Web Services, Journal of Medical Systems archive, Volume 38 Issue 4, April 2014, Pages 1-14; Plenum Press New York, NY, USA, http://dx.doi.org/10.1007/s10916-014-0032-2

[10] Giovanni Vigna, Fredrik Valeur, Richard A. Kemmere, "Designing and implementing a family of intrusion detection systems”, CM SIGSOFT Software Engineering, Vol 28 Issue 5, September 2003, Pages 88-97, http://dx.doi.org/10.1145/949952.940084

[11]El Mabrouk Marouane, Cherrat Loubna, Ezziyyani Mostafa and Essaaidi Mohammad, "Conception of Real-Time Flood Forecasting System Approach Based on Anytime Techniques, in Proceeding Engineering \& Technology (PET) of the International Conference on Control, Engineering \& Information Technology (CEIT’13)”, Vol.1, pp. 207-211, 2013.

[12]Ian P. McCarthy, Christos Tsinopoulos, Peter Allen ${ }^{3}$ and Christen Rose-Anderssen, "New Product Development as a Complex Adaptive System of Decisions” Journal of Product Innovation Management, Volume 23, Issue 5, Pages 437- 456, September 2006 http://dx.doi.org/10.1111/j.1540-5885.2006.00215.x.

[13]Rouse, W.B. 2006. Agile Information Systems for Agile Decision Making. Chapter 2 in Agile Information Systems, edited by K.C. Desouza. New York: Butterworh- Heinemann.

[14]T. INAGAKI “Situation-adaptive autonomy for time-critical takeoff decisions", International journal of modelling \& simulation, Volume 20, no2, pages 175-180, 2000. http://dx.doi.org/10.1109/TSMCA.2009.2026428

[15]Saporta G., "Probabilités, analyse des données et statistique”, 2eme édition. Editions Technip, 656 pages, 2006.

[16]Andrew G. Barto , R. S. Sutton , C. J. C. H. Watkins "Learning and Sequential Decision Making” Journal Artificial Intelligence Vol 72 Issue 1-2, January, 1995 p 81-138 http://dx.doi.org/10.1016/0004-3702(94)00011-O

\section{Copyright Disclaimer}

Copyright reserved by the author(s).

This article is an open-access article distributed under the terms and conditions of the Creative Commons Attribution license (http://creativecommons.org/licenses/by/3.0/). 